\title{
Análise da força isométrica máxima e do sinal de EMG em exercícios para os membros inferiores
}

\author{
Analysis of maximal isometric force and EMG signal \\ in lower limb exercise
}

\author{
Cleiton Silva Correa \\ Bruna Gonçalves Cordeiro da Silva \\ Cristine Lima Alberton 1 \\ Eurico Nestor Wilhelm 1 \\ Antonio Carlos de Moraes 2 \\ Cláudia Silveira Lima \\ Ronei Silveira Pinto 1
}

1 Universidade Federal do Rio Grande do Sul. Laboratório de Pesquisa do Exercício. Porto Alegre, RS. Brasil.

2 Universidade Estadual de Campinas. Campinas. São Paulo, SP. Brasil

Recebido em 02/03/11 Revisado em 15/03/11 Aprovado em 09/05/11
Resumo - O objetivo deste estudo foi comparar a força isométrica máxima (FIM) e a atividade elétrica dos músculos vasto medial (VM), vasto lateral (VL), reto femoral (RF), glúteo máximo (GM) e bíceps femoral (BF) entre contrações isométricas voluntárias máximas (CIVM) executadas em diferentes posições articulares e identificar as mais adequadas para normalizar o sinal de eletromiografia (EMG) de cada um dos músculos referidos, quando estes são ativados em condições dinâmicas. A amostra foi constituída de 10 indivíduos do sexo masculino, com idades entre 20 e 30 anos, familiarizados com treinamento de força. Foram realizadas CIVMs em diferentes ângulos articulares para os grupos extensores e flexores do joelho $\left(0^{\circ}, 60^{\circ}, 90^{\circ}\right)$ e extensores $\left(-30^{\circ}, 0^{\circ}, 60^{\circ}\right)$ e flexores do quadril $\left(90^{\circ}, 120^{\circ}\right)$. Sobre a FIM foram encontrados valores significativamente diferentes entre as posições em $60^{\circ}$ para flexão de joelhos e para extensão de joelhos em $60^{\circ}$ e $90^{\circ} \quad(p<0,01)$ e não houve diferenças significativas entre as posições na flexão e extensão do quadril (p>0,05). Em relação ao sinal EMG, foram encontrados valores significativamente diferentes entre os ângulos para a extensão de joelhos somente no $\mathrm{RF}$ em $90^{\circ}$ ( $\left.\mathrm{p}<0,01\right)$. Para a flexão de joelhos, flexão e extensão de quadril não foram encontradas diferenças siginificativas entre os músculos nos diferentes ângulos avaliados $(\mathrm{p}>0,05)$. Estes resultados sugerem que a posição de $60^{\circ}$ é a mais adequada para avaliação dos flexores e extensores do joelho e que todas posições testadas neste estudo apresentam-se como adequadas para a avaliação da flexão e extensão de quadril.

Palavras-chave: Contração muscular; Eletromiografia; Força muscular; Joelho; Quadril.

Abstract - The aim of this study was to compare maximal isometric force (MIF) and the electrical activity of the vastus medialis, vastus lateralis, rectus femoris, gluteus maximus and biceps femoris long head muscles between maximal voluntary contractions (MVC) performed at different joint angles, and to identify the most suitable positions to normalize the electromyography (EMG) signals from each of these muscles when they are activated under dynamic conditions. Ten men ranging in age from 20 to 30 years, who were familiar with strength training exercise, were studied. MVC at different joint angles of the knee extensors and flexors $\left(0^{\circ}, 60^{\circ}, 90^{\circ}\right)$ and hip extensors $\left(-30^{\circ}, 0^{\circ}, 60^{\circ}\right)$ and flexors $\left(90^{\circ}, 120^{\circ}\right)$ were tested. The MIF values differed significantly between the $60^{\circ}$ knee flexion and $60^{\circ}$ and $90^{\circ}$ knee extension positions $(p<0.01)$. The same was not observed for hip flexion or extension ( $p>0.05)$. Significantly higher EMG values were only observed for the rectus femoris muscle at $90^{\circ}$ knee extension $(p<0.01)$. No differences between muscles were found for knee flexion, hip flexion or hip extension at the joint angles tested ( $p>0.05$ ). These results suggest that the $60^{\circ}$ knee joint flexion position is the most suitable for assessment of knee extension and flexion MIF, and that all positions tested in this study are suitable for the assessment of hip flexion and extension.

Key words: Electromyography; Hip; Knee; Muscle contraction; Muscle strength. 


\section{INTRODUÇÃO}

A eletromiografia $(E M G)$ pode ser definida como um método de registro dos potenciais elétricos gerados nas fibras musculares, durante os diferentes tipos de contração, e possibilita a avaliação do comportamento do sistema neuromuscular. A EMG de superfície tem sido usada principalmente para detectar e analisar a atividade mioelétrica de músculos específicos em determinados tipos de movimento ${ }^{1-6}$.

Para a correta interpretação dos dados relativos à atividade elétrica dos músculos analisados, a normalização do sinal de EMG é essencial para as comparações entre diferentes dias de coleta, músculos analisados, estudos e, principalmente, entre os indivíduos, numa tentativa de minimizar as diferenças existentes relacionadas à estatura, massa corporal, massa muscular, nível de treinamento e ainda, outros aspectos ${ }^{7,8}$. Diferentes padrões têm sido adotados para a normalização do sinal de EMG, sendo mais frequente a utilização do valor máximo obtido em contrações isométricas voluntárias máximas (CIVMs) ${ }^{9,10}$. No entanto, há significativa diferença na produção de força e, consequentemente, do sinal de EMG dos músculos ativados, entre as diferentes posições articulares em que os testes isométricos são realizados ${ }^{11} \mathrm{e}$ ainda não são conhecidos quais os ângulos articulares mais adequados para a obtenção dos valores de sinal EMG máximos para serem utilizados para posterior normalização dos outros sinais.

Neste sentido, a determinação do ângulo articular em que ocorre a maior vantagem mecânica muscular e, possivelmente, o maior valor representativo da atividade miolétrica, assume importância metodológica no estudo dos níveis de ativação de músculos específicos. Sobretudo, para a determinação de critérios de normalização do sinal de EMG desses músculos que são solicitados em diferentes tipos de exercício e movimentos corporais ${ }^{12-14}$.

Não é de nosso conhecimento a existência de estudos realizados com o objetivo de investigar e comparar quais ângulos articulares são mais adequados para a avaliação da força e da ativação muscular. Sendo essa uma questão de significativa importância metodológica para a realização de estudos da área, o objetivo do presente estudo foi determinar em quais ângulos articulares observam-se maiores níveis de produção de força isométrica máxima (FIM), bem como maiores níveis de atividade elétrica dos músculos reto femoral (RF), na extensão isométrica de joelho e na flexão isométrica de quadril, vasto lateral (VL) e vasto medial (VM), na extensão isométrica de joelho, bíceps femoral cabeça longa (BF), na flexão isométrica de joelho, bem como do glúteo máximo (GM), na extensão isométrica de quadril.

\section{PROCEDIMENTOS METODOLÓGICOS}

A amostra foi constituída por 10 voluntários saudáveis do sexo masculino $(22,80 \pm 3,42$ anos; $177,4 \pm$ $5,75 \mathrm{~cm} ; 78,9 \pm 9,40 \mathrm{~kg} ; 12,4 \pm 6,9 \%$ de gordura) com, pelo menos, um ano de prática em treinamento de força e sem histórico de lesão nas articulações do joelho e do quadril. O cálculo do "n" amostral foi realizado no programa PEPI versão 4.0, com base nas médias e desvios-padrão dos estudos de Da Silva et al. ${ }^{12}$ e Escamilla et al. ${ }^{13}$, adotando-se um poder de $90 \%$. Todos os indivíduos assinaram um termo de consentimento livre e esclarecido. Esta pesquisa foi aprovada pelo Comitê de Ética em Pesquisa da Universidade Federal do Rio Grande do Sul (2008/012).

Os sujeitos compareceram à Escola de Educação Física (ESEF) da Universidade Federal do Rio Grande do Sul (UFRGS), em dois diferentes dias, para a coleta de dados. No primeiro dia, foram apresentados os objetivos e detalhes metodológicos do estudo e foi assinado o termo de consentimento informado, livre e esclarecido. Posteriormente, foi realizada a familiarização dos sujeitos com os testes. No segundo dia, foram realizadas as medidas antropométricas e a aquisição do sinal de EMG nos diferentes ângulos articulares, de flexão e extensão do joelho e de flexão e extensão do quadril, nas quais os músculos investigados (VL, RF, VM, BF e GM) atuam como motores primários.

\section{Protocolo}

As CIVMs, em cada uma das posições avaliadas, foram realizadas durante um período de cinco segundos $\mathrm{cada}^{2}$, durante o qual foram simultaneamente captados os sinais de força e o sinal de EMG dos músculos RF, VL e VM na extensão isométrica de joelho, do BF na flexão isométrica do joelho, do BF na flexão isométrica do joelho e na extensão do quadril, bem como do GM na extensão isométrica do quadril e do RF na flexão isométrica de quadril nos diferentes ângulos articulares avaliados. Foram realizadas três CIVMs em cada ângulo, com o membro dominante, determinado como a perna de chute do sujeito, e foi dado um intervalo de cinco minutos entre os testes. Durante a execução dos testes, os sujeitos foram estimulados com encorajamento verbal a produzirem a máxima força possível. 
Na extensão isométrica de joelho (CIVM), os sujeitos foram posicionados sentados, com o quadril em $110^{\circ}$ e o joelho em $0^{\circ}$ (extensão completa do joelho), $60^{\circ}$ e $90^{\circ}$ para os testes. As CIVMs de flexão do joelho foram realizadas da mesma maneira e também com o joelho nos ângulos de $0^{\circ}, 60^{\circ} \mathrm{e}$ $90^{\circ}$. Estas CIVMs foram realizadas em um equipamento de extensão de joelhos (Sculptor, Porto Alegre, Brasil), com as cargas do equipamento fixadas de maneira a impedir o seu deslocamento, tendo sido acoplada uma célula de carga (CC) (Miotec, Equipamentos Biomédicos, Brasil), com resolução de $1 \mathrm{kgf}$, ao cabo do equipamento, a qual possibilitou avaliar a força de tração desenvolvida durantes estas CIVMs. A CC estava conectada a um conversor análogico digital (A/D) Miotool (Miotec, Equipamentos Biomédicos, Brasil).

As CIVMs de extensão do quadril foram realizadas na posição em pé, com os joelhos em $0^{\circ} \mathrm{e}$ com os braços apoiados num espaldar para auxiliar no equilíbrio e a articulação do quadril foi então posicionada em $-30^{\circ}, 0^{\circ}$ e $60^{\circ}$ e a CC foi acoplada ao espaldar e a uma cinta fixada no tornozelo dos sujeitos. As CIVMs de flexão do quadril foram realizadas na posição sentada com o joelho em $90^{\circ}$ e o quadril em $90^{\circ}$ e $120^{\circ}$ e a CC foi acoplada em uma estrutura de metal fixa ao solo e a uma cinta posicionada na coxa dos sujeitos.

\section{Posicionamento dos eletrodos}

Para a coleta do sinal de EMG dos músculos avaliados, foram utilizados eletrodos de superfície (Tyco Healthcare, Mini Medi-Trace 100, Kendall Medtrace), com $15 \mathrm{~mm}$ de raio, sendo dispostos em configuração bipolar com 20 mm de distância intereletrodos.

Antes da colocação dos eletrodos, realizou-se a tricotomia e raspagem da pele seguida de abrasão com algodão e álcool. Esse procedimento foi realizado para retirar as células mortas e diminuir a impedância da pele no local em que foram colocados. A seguir, os eletrodos foram posicionados no ventre dos músculos avaliados, conforme proposto por Leis e Trapani ${ }^{15}$.

A impedância intereletrodos manteve-se abaixo de 3000 Ohms antes do início de cada coleta ${ }^{16}$, avaliada por multímetro digital. O eletrodo de referência foi posicionado no olécrano da ulna.

\section{Aquisição do sinal de EMG e de Força}

Para a aquisição dos sinais de EMG e da CC, foi utilizado um eletromiógrafo Miotool 400 (Miotec, Equipamentos Biomédicos, Brasil), composto por um sistema de quatro canais que opera em uma taxa de aquisição fixa de $2000 \mathrm{~Hz}$ por canal.

\section{Tratamento dos sinais de FIM e EMG}

Os sinais capturados pelo sistema de EMG foram armazenados no software Miograph (versão 2.0.20), para posterior análise no software SAD32. Para o tratamento dos sinais de FIM, foi utilizado um filtro do tipo passa-banda Butterworth com frequência de corte 0 e $9 \mathrm{~Hz}$, de quinta ordem ${ }^{17}$. Os valores de maior magnitude de FIM e de sinal de EMG para cada músculo foram utilizados para a comparação entre os diferentes ângulos. Os sinais de força de cada CIVM foram tratados em intervalos de, aproximadamente, um segundo, quando se apresentavam em condições próximas do máximo e com reduzida oscilação da curva (platô). Os sinais EMG, sincronizados ao platô dos sinais de força, foram, inicialmente, filtrados a partir de um filtro do tipo passa-banda Butterworth, de quinta ordem, com frequência de corte de 20 a $450 \mathrm{~Hz}$. Posteriormente, a magnitude do sinal de EMG foi calculada em valores de root mean square (RMS) em milivolts $(\mathrm{mV})$ calculados em um período de, aproximadamente, um segundo ${ }^{17}$ sincronizados ao platô da curva de força ${ }^{12,17,18}$.

\section{Análise Estatística}

Para a análise dos dados coletados, foi utilizada a estatística descritiva (média \pm desvio-padrão), sendo que a sua normalidade foi verificada pelo teste de Shapiro-Wilk. A comparação do nível de ativação muscular dos músculos monitorados e a FIM, ambas mensuradas nos diferentes ângulos, foi realizada a partir da ANOVA para medidas repetidas. Utilizou-se post hoc de Bonferroni para a localização das comparações múltiplas. O nível de significância utilizado em todos os procedimentos estatísticos foi de $\alpha<0,05$, sendo estes realizados no software SSPS versão 15.0.

\section{RESULTADOS}

\section{Força Isométrica Máxima}

Os valores de FIM relativos às CIVMs de extensão de joelhos foram estatisticamente semelhantes entre os ângulos de $60^{\circ}$ e $90^{\circ}$, sendo estes significativamente superiores (aproximadamente 34\% e $39 \%$, respectivamente) dos valores registrados no ângulo de $0^{\circ}(\mathrm{p}<0,01)$. Na flexão de joelhos, os valores de FIM nas CIVMs realizadas em $0^{\circ} \mathrm{e}$ $90^{\circ}$ foram semelhantes, e estes significativamente menores (aproximadamente $46 \%$ e 31\%, respec- 
tivamente) do que os valores observados em $60^{\circ}$ $(\mathrm{p}<0,01)$ (Figura 1). Por outro lado, os valores de FIM relativos às CIVMs de extensão e flexão de quadril não apresentaram diferenças entre os ângulos avaliados ( $>>0,05)$ (Figura 2).

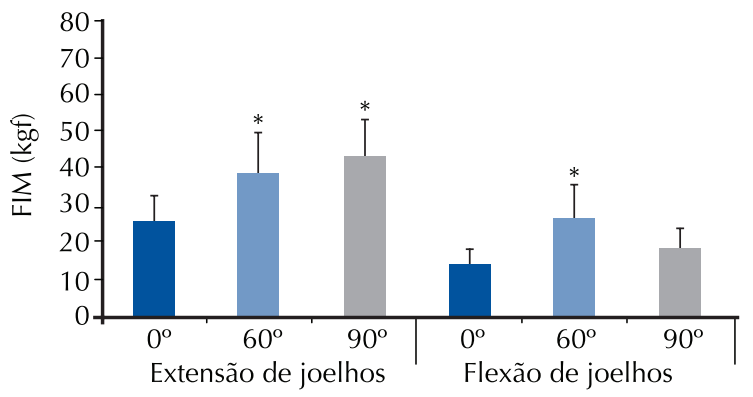

Figura 1. Valores de força isométrica máxima de extensão e flexão de joelhos (média \pm DP) nos diferentes ângulos; $(*)$ valores significativamente maiores $(p<0,01)$ do que nos demais ângulos avaliados.

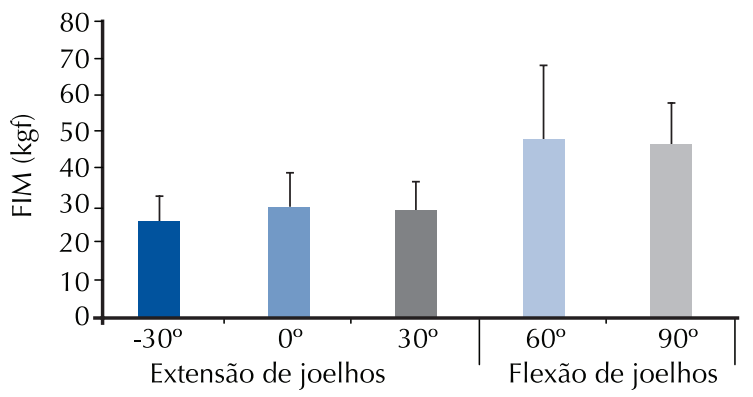

Figura 2. Valores de força isométrica máxima de extensão e flexão de quadril (média \pm DP) nos diferentes ângulos

\section{Sinal de EMG}

Os valores relativos ao sinal de EMG dos músculos VM e VL durante as CIVMs de extensão de joelho não apresentaram diferença estatisticamente significativa nos diferentes angulos avaliados $(\mathrm{p}>0,05)$. No entanto, o músculo RF foi significativamente mais ativado $(\mathrm{p}=0,013)$ nas CIVMs de extensão do joelho executadas no ângulo de $90^{\circ}$ do que nos ângulos de $0^{\circ}$ e $60^{\circ}$ (Figura 3).

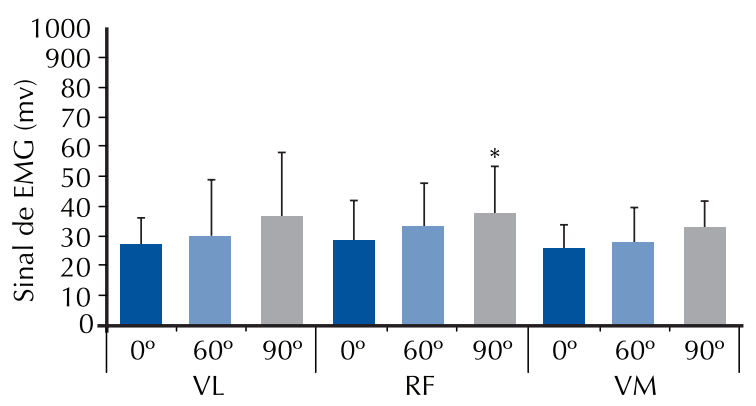

Figura 3. Valores de RMS (mV) (média $\pm \mathrm{DP})$ dos músculos VL (vasto lateral), RF (reto femoral) e VM (vasto medial) na extensão isométrica de joelhos em $0^{\circ}, 60^{\circ}$ e $90^{\circ} ;\left(^{*}\right)$ valores significativamente maiores $(p<0,01)$ do que nos demais ângulos avaliados $\left(0^{\circ}\right.$ e $\left.60^{\circ}\right)$.

Os valores relativos ao sinal de EMG do mús- culo BF, durante as CIVMs de flexão de joelho, não apresentaram diferença entre os ângulos $(\mathrm{p}>0,05)$ (Figura 4), o mesmo ocorrendo com o músculo GM, durante as CIVMs de extensão do quadril, em todos os ângulos avaliados e com o RF durante as flexões do quadril realizadas $(\mathrm{p}>0,05)$ (Figura 5).

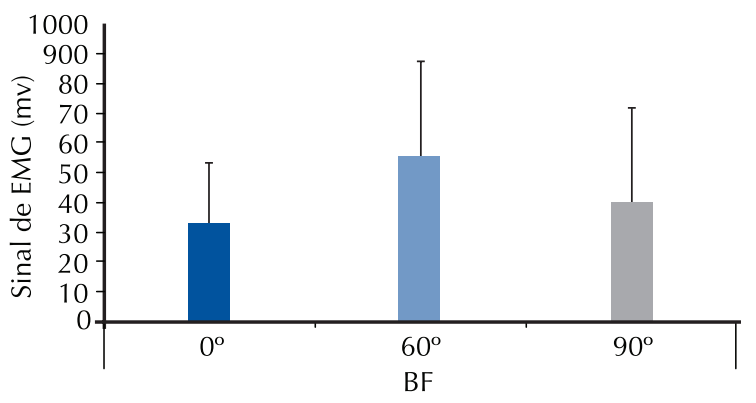

Figura 4. Valores de RMS $(\mathrm{mV})$ (média $\pm \mathrm{DP}$ ) do músculo BF (bíceps femoral) na flexão isométrica de joelhos em $0^{\circ}, 60^{\circ}, 90^{\circ}$.

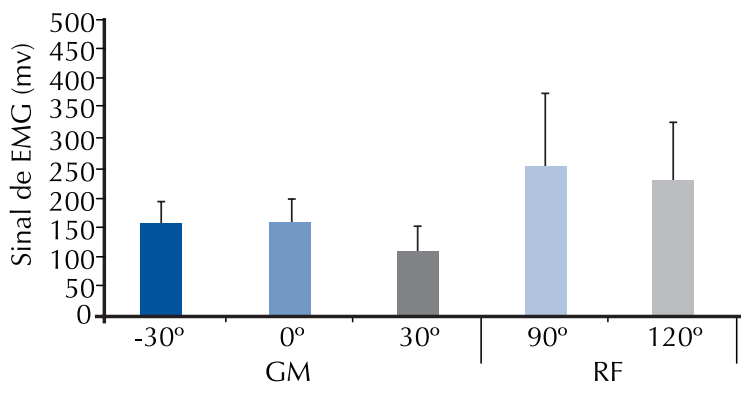

Figura 5. Valores de RMS (mV) (média \pm DP) do músculo GM (glúteo máximo) na extensão isométrica de quadril em $-30^{\circ}$, $0^{\circ}$ e $60^{\circ}$, e do músculo RF (reto femoral) na flexão isométrica de quadril em $90^{\circ}$ e $120^{\circ}$.

\section{DISCUSSÃO}

Os principais achados do presente estudo indicaram diferença significativa entre os ângulos articulares nos valores de FIM na extensão e flexão de joelhos e no sinal de EMG do músculo RF na extensão isométrica de joelho. Os valores da força muscular na extensão de joelho demonstraram que os ângulos de $60^{\circ}$ e $90^{\circ}$ são semelhantes entre si quanto à produção de força isométrica, sendo superiores em relação a $0^{\circ}$. Na flexão de joelhos, o ângulo de $60^{\circ}$ apresentou maiores valores de FIM que o de $90^{\circ}$ e $0^{\circ}$.

Esses resultados demonstram que a máxima produção de força isométrica ocorre a $60^{\circ}$ e $90^{\circ}$ para a extensão de joelhos e a $60^{\circ}$ para a flexão de joelhos. Diversos autores utilizam o ângulo de $60^{\circ}$ ou ângulos próximos a ele para avaliar a produção de força máxima dos extensores e flexores do joelho ${ }^{8,10}$. Já Higashihara et al. ${ }^{19}$, embora tenham avaliado a ativação do BF durante a flexão excêntrica de joelho, em diferentes velocidades, encontraram, também, em ângulos próximos a $60^{\circ}$ 
uma maior ativação deste músculo. Embora alguns autores ${ }^{20}$ tenham utilizado o ângulo de $90^{\circ}$ para a mensuração das CIVMs de flexão e extensão de joelhos, os demais estudos supracitados, bem como o presente, apontam o ângulo de $60^{\circ}$ como aquele em que há grande vantagem mecânica muscular tanto para extensão quanto para a flexão de joelhos. Essa vantagem faz com que o ângulo de $60^{\circ}$ possa ser considerado um ângulo de ótima produção de força de flexão de joelho enquanto que os ângulos de $60^{\circ}$ e $90^{\circ}$ também parecem adequados para a extensão de joelhos.

Além disso, a FIM não diferiu entre os ângulos avaliados na flexão e extensão de quadril, indicando que qualquer uma dessas angulações podem ser utilizadas para a aquisição da FIM de extensores e flexores do quadril.

Em diversos estudos, nos quais a coleta do sinal de EMG, durante a extensão de joelhos, foi realizada, os músculos VL, RF e VM foram utilizados para mensurar a ativação muscular ${ }^{8,13,21,22}$. No presente estudo, o VM não apresentou diferença no sinal de EMG nos ângulos avaliados. No entanto, Pincivero et $a .^{10}$ reportaram que a máxima ativação do VM ocorre nos ângulos próximos à máxima extensão de joelho e em níveis máximos de força voluntária, nos quais a atividade eletromiográfica torna-se equivalente a dos músculos VL e RF. Alkner et al..$^{23}$ sugerem que o músculo VM é o mais ativado em níveis próximos do máximo da CIVM e nos ângulos próximos da máxima extensão de joelhos, já no presente estudo não foram encontradas diferenças entre a ativação do VM nos ângulos de 0, 60 e $90^{\circ}$, provavelmente, por diferenças metodológicas. Com relação ao VL, este músculo não apresentou diferença significativa de ativação nos diferentes ângulos, e não foram encontrados estudos com os quais se possa comparar os resultados do presente estudo. Contudo, assim como o VM, o VL é um constituinte monoarticular do quadríceps femoral e possivelmente, apresentaria valores de ativação máxima em ângulos semelhantes aos do VM.

Dos três ventres do quadríceps femoral que foram avaliados em cadeia cinética aberta (CCA), o RF foi o único que apresentou diferença significativa entre os ângulos na CIVM de extensão de joelho. Para Akima et al. ${ }^{24}$, o recrutamento do RF não necessariamente coincide com os três vastos durante exercícios isométricos e isocinéticos. Isto pode ser explicado pelo fato do RF ser biarticular e ter função de distribuir torque para ambas articulações do quadril e joelho, considerando que os três vastos são sinergistas quando agem somente na ar- ticulação do joelho. Estes resultados corroboraram o estudo de Escamila et al. ${ }^{21}$, que observaram maior ativação do VM e VL (20\% e 5\%, respectivamente), durante a extensão de joelhos em exercícios de cadeia cinética fechada (CCF). Por outro lado, o $\mathrm{RF}$ foi significativamente mais ativado na extensão de joelho (45\%) em CCA do que nos exercícios de CCF. Já, como flexor do quadril nenhuma diferença foi encontrada nas angulações de $90^{\circ}$ e $120^{\circ}$ de flexão de quadril, provavelmente, devido ao comprimento muscular ter sido muito pouco afetado, diferentemente do ocorrido na extensão de joelhos.

Ainda com relação aos extensores de joelho e sua ativação, Watanabe e Akima ${ }^{14}$ encontraram que o RF foi o músculo mais ativado do quadríceps femoral durante uma extensão isométrica de joelho a $80 \%$ da CIVM. Rabita et al. ${ }^{8}$ encontraram maior ativação do RF em relação ao VM e VL tanto pré quanto pós-período de treinamento de força. Signorile et al..$^{25}$ reportaram que a atividade elétrica do VM e VL é maior no agachamento do que na extensão de joelho para uma carga de mesma intensidade relativa. Por outro lado, no presente estudo, não foram encontradas diferenças significativas na ativação muscular entre os músculos do quadríceps femoral, possivelmente, pela condição isométrica utilizada, a qual reduz a influência do encurtamento muscular e consequente deslocamento das fibras musculares e do ponto motor em relação aos eletrodos. Alguns autores ${ }^{14}$ ainda referem que estas diferenças entre os resultados de estudos podem ser atribuídas a outras condições experimentais.

Com relação à ativação do VL na extensão de joelho, sugere-se $\mathrm{s}^{10,23}$ que este constitui o músculo mais representativo do quadríceps femoral quando se trata da quantificação das alterações neurais produzidas pelo treino de força, principalmente por apresentar menor variabilidade intraindividual do sinal de EMG. No presente estudo, foi observada variação considerável (elevado desvio padrão) do sinal de EMG desse músculo. No entanto, isto ocorre, pois os valores de EMG são apresentados de forma absoluta, uma vez que foram coletados apenas em CIVMs e, por isso, sem normalização. Acredita-se que os valores de sinal de EMG do VL apresentariam menor variabilidade, como sugerido pelos autores acima, caso tivessem sido coletados também em contrações dinâmicas e normalizados pela ativação durante as CIVMs.

Já o flexor do joelho BF cabeça longa constitui-se em um músculo biarticular ${ }^{26}$. Alguns estudos utilizam apenas o BF para análise de sinal de EMG, demonstrando este músculo ser representativo dos 
isquitibiais ${ }^{12,27}$. No presente estudo, nenhuma diferença significativa foi encontrada na ativação deste músculo nos diferentes ângulos avaliados. Não é de nosso conhecimento a existência de outro estudo que tenha avaliado e comparado a ativação do BF em diferentes ângulos na flexão isométrica de joelhos.

Quanto ao músculo GM, não foi encontrada diferença significativa entre os ângulos avaliados de extensão de quadril. Caterisano et al..$^{28}$, investigaram a participação do GM a partir do pico de sinal de EMG, em condições dinâmicas, durante as fases inicial, intermediária e final do exercício de agachamento (exercício em CCF), encontraram diferença significativa no pico do sinal de EMG do GM na fase final. Sabe-se que quando o encurtamento muscular é aumentado, maior é o recrutamento de unidades motoras auxiliares para a produção de FIM, o que decorre em incremento do sinal de $\mathrm{EMG}^{12,29}$. Esses achados contrapõem o estudo de Newman et al. ${ }^{20}$, o qual não refere diferença no sinal de EMG em diferentes comprimentos articulares de membros inferiores, bem como os resultados do presente estudo. Acredita-se que essas discrepâncias entre os achados estejam relacionadas com as diferentes metodologias utilizadas entre os estudos.

Uma limitação do presente estudo pode ter sido a presença de uma polia excêntrica no equipamento de extensão de joelho, o que supostamente corrigiria as alterações biomecânicas que afetariam a capacidade de produção de força. No entanto, é sugerido que nem sempre os equipamentos de resistência variável correspondem à capacidade de produção de força muscular ao longo dos ângulos $\operatorname{articulares}^{30}$. No presente estudo, a relação torque-ângulo não foi avaliada no equipamento utilizado; desta forma, os valores obtidos nos diferentes ângulos avaliados de extensão e flexão de joelhos podem ter sido afetados pela presença da polia excêntrica do equipamento.

\section{CONCLUSÕES}

O presente estudo demonstrou que os valores de FIM podem ser adequadamente adquiridos nos ângulos de $90^{\circ}$ e $60^{\circ}$, nas CIVMs de extensão de joelhos, e $60^{\circ}$ nas CIVMs de flexão de joelhos. De maneira geral, pode-se concluir que o ângulo de $60^{\circ}$ tanto de extensão quanto de flexão de joelhos apresenta a maior vantagem mecânica muscular em relação à FIM e ao sinal de EMG dos músculos, e constitui-se na angulação mais adequada, em termos metodológicos, para a avaliação destas variáveis. Para a flexão e extensão de quadril, os valores de FIM e de sinal de EMG dos músculos RF e GM podem ser mensurados em qualquer uma das angulações testadas no presente estudo. No que se refere à máxima atividade eletromiográfica dos três ventres do quadriceps avaliados (VL, VM, RF), durante a extensão isométrica de joelhos, os valores dos músculos VL e VM mostram que estes podem ser avaliados nos ângulos $0^{\circ}, 60^{\circ}$ e $90^{\circ}$, uma vez que têm comportamento semelhante nestes ângulos. Já os valores do RF mostram o ângulo de $90^{\circ}$ como o mais adequado para avaliar a sua ativação máxima.

\section{REFERÊNCIAS BIBLIOGRÁFICAS}

1. Basmajian JV, De Luca CJ. Muscles alive: their functions revealed by electromyography. Baltimore: Williams and Wilkins; 1985.

2. De Luca CJ. The use of surface electromyography in biomechanics. J Appl Biomech 1997;13:135-63.

3. Macdonald JH, Farina D, Marcora SM. Response of Electromyography Variables during Incremental and Fatiguing Cycling. Med Sci Sports Exerc 2008;40:335-44.

4. Onishi H, Yagi R, Akasaka K, Momose K, Ihashi K, Handa Y. Relationship between EMG signals and force in human vastus lateralis muscle using multiple bipolar wire electrodes. J Electromyogr Kinesiol 2000;10:59-67.

5. Soderberg GL, Knutson LN. A Guide for Use and Interpretation of Kinesiology Electromyographic Data. Phys Ther 2000;80(5):585-98.

6. Stashuk D. EMG signal decomposition: how can it be accomplished and used? J Electromyogr Kinesiol 2001;151-73.

7. Beck TW, Housh TJ, Cramer JT, Weir JP. The Effect of the Estimated Innervation Zone on EMG Amplitude and Center Frequency. Med Sci Sports Exerc 2007;39:1282-90.

8. Rabita G, Pérot C, Lensel-Corbeil G. Differential effect of knee extension isometric training on the different muscles of the quadriceps femoris in humans. Eur J Appl Physiol 2000;83:531-8.

9. Kellis E, Baltzopoulos V. The effects of normalization method on antagonistic activity patterns during eccentric and concentric isokinetic knee extension and flexion. J Electromyogr Kinesiol 1996;6:235-45.

10. Pincivero DM, Coelho AJ, Campy RM, Salfetnikov Y, Suter E. Knee extensor torque and quadriceps femoris EMG during perceptually-guided isometric contractions. J Electromyogr Kinesiol 2003;13(2):159-67.

11. Murphy AJ, Wilson GJ. Poor correlations between isometric tests and dynamic performance: relationship to muscle activation. Eur J Appl Physiol 1996;73:353-7.

12. Da Silva EM, Brentano AM, Cadore EL, De Almeida APV, Kruel LFM. Analysis of muscle activation during different leg press exercise at submaximum effort levels. J Strength Cond Res 2008;22:1059-65.

13. Escamilla RF, Fleisig GS, Zheng N, Lander JE, Bar- 
rentine SW, Rews JR, Bergemann BW, Moorman CT. Effects of technique variations on knee biomechanics during squat and leg press. Med Sci Sports Exerc 2001;3:1552-66.

14. Watanabe K, Akima H. Normalized EMG to normalized torque relationship of vastus intermedius muscle during isometric knee extension. Eur J Appl Physiol 2009;106:665-73.

15. Leis AA, Trapani VC. Atlas of electromyography. Oxford, NY: Oxford University Press; 2000.

16. Narici M, Vroi GS, Landoni L, Minetti AE, Cerretelli P. Changes in force, cross-sectional area and neural activation during strength training and detraining of the human quadriceps. Eur J Appl Physiol 1989;59:310-9.

17. Cadore EL, Pinto RS, Alberton CL, Pinto SS, Lhullier FL, Tartaruga MP, et al. Neuromuscular Economy, Strength, and Endurance in Healthy Elderly Men. J Strength Cond Res 2011;25(4):997-1003.

18. Kalmar JM, Cafarelli E. Central excitability does not limit post fatigue voluntary activation of quadriceps femoris. J Appl Physiol 2006;100:1757-64.

19. Higashihara A, Ono T, Kubota J, Fukubayashi T. Differences in the electromyographic activity of the hamstring muscles during maximal eccentric knee flexion. Eur J Appl Physiol 2010;108:355-62.

20. Newman SA, Jones G, Newham DJ. Quadriceps voluntary activation at different joint angles measured by two stimulation techniques. Eur J Appl Physiol 2003;89:496-9.

21. Escamilla RF, Fleisig GS, Zheng N, Barrentine SW, Andreews JR. Biomechanics of the knee during cloud kinetic chain open kinect chain exercise. Med Sci Sports Exerc 1998;30:556-9.

22. Häkkinen K, Komi PV. Electromyography changes during strength training and detraining. Med Sci Sports Exerc 1983;15:455-60.

23. Alkner BA, Tesch PA, Berg HE. Quadriceps EMG/force relationship in knee extension and leg press. Med Sci Sports Exerc 2000;32:459-63.

24. Akima H, Takahashi H, Kuno S, Katsuta S. Coacti- vation pattern in human quadriceps during isokinetic knee-extension by muscle functional MRI. Eur J Appl Physiol 2004;91:7-14.

25. Signorile JF, Weber B, Roll B, Caruso JF, Lowensteyn I, Perry AC. An electromyographical comparison of the squat and knee extension exercises. J Strength Cond Res 1994;8:178-83.

26. Onishi H, Yagi R, Oyama M, Akasaka K, Ihashi K, Handa Y. EMG-angle relationship of the hamstrings muscles during maximum knee flexion. J Electromyogr Kinesiol 2002;12:399-406.

27. Ebben WP. Hamstring activation during lower body resistance training exercises. Int J Sports Physiol Perform 2009;4(1):84-96.

28. Caterisano A, Moss RE, Pellinger TK, Woodruff K, Lewis VC, Booth W, et al. The effect of back squat depth on the EMG activity of 4 superficial hip and thigh muscles. J Strength Cond Res 2002;16:428-32.

29. Chilibeck PD, Calde AW, Sale DG, Webber CE. A comparison of strength and muscle mass increases during resistance training in young women. Eur J Appl Physiol 1998;77:170-5.

30. Folland J, Morris B. Variable-cam resistance training machines: Do they match the angle - torque relationship in humans? J Sports Sci 2008; 26:163-9. 